\title{
ON THE MEASURE OF THE STRUCTURE AROUND AN INVARIANT KAM TORUS
}

ANALYTICAL AND NUMERICAL INVESTIGATION .

\author{
C.FROESCHLÉ ${ }^{1}$, A.GIORGILLI $^{2}$, E.LEGA ${ }^{1,3}$, A.MORBIDELLI $^{1}$ \\ 1 Observatoire de Nice B.P.229, 06304 Nice cedex 4 \\ ${ }^{2}$ Dipartimento di Fisica dell'Università, Via Celoria 16, Milan \\ ${ }^{3}$ LATAPSES, 250 Rue A.Einstein, 06560 Valbonne
}

\section{Introduction}

In a recent paper, Morbidelli and Giorgilli (1995) proved the superexponential stability of invariant tori. As usual in the theory of dynamical systems, the results are rigorously proved assuming that the perturbation is small enough. The numerical experiments show, however, that invariant tori persist up to much larger perturbation magnitudes. Therefore, it is interesting to check numerically if the superexponential stability and the other properties outlined in Morbidelli and Giorgilli's theorem persist up to the value of the perturbation for which the torus actually breaks up. Moreover, one would like to have a numerical indication about the size of the superexponentially stable region existing around a torus. Is the superexponential stability just an asymptotic result, or does it concern a macroscopic region of physical interest?

The relation of this work with the dynamics of the solar system may not appear clear at a first glance. Nevertheless, it is well known that the fundamental problem of the stability of the solar system is connected with the stability of non linear Hamiltonian systems. At the beginning of this century Poincaré had already shown that it was impossible to demonstrate the integrability of the Hamiltonian system representing the motion of the solar system. In 1954 Kolmogorov obtained a partial integrability, for some small values of the perturbing parameter $\epsilon$, for Hamiltonian systems described by:

$$
H(p, q)=H_{0}(p)+\epsilon H_{1}(p, q)
$$

The next step is due to the work of Nekhoroshev (1977) concerning the diffusion of the actions of the invariant KAM tori. Although the mathematical 
demonstrations require small perturbations (direct application implies that the Jupiter mass is much smaller than the size of an orange), the numerical experiments (Hénon 1969) have shown the existence of the regions of stability up to larger perturbation magnitudes.

This work has been realized, respect to the Morbidelli and Giorgilli's theorem, in the same spirit of that of Hénon respect to the KAM theorem. The aim is therefore to be in continuity with the researches about the stability of Hamiltonian systems, and hence of the solar system.

Our numerical computations show in a striking way that the description of the dynamics given, for small perturbations, by Morbidelli and Giorgilli's result is true in reality as long as the invariant torus persists. Moreover, the size of the structure described by Morbidelli and Giorgilli around the invariant torus shrinks to 0 like $\exp \left(-\epsilon_{c} /\left(\epsilon_{c}-\epsilon\right)\right)$ when the size of the perturbation $\epsilon$ tends to the threshold value $\epsilon_{c}$ corresponding to the torus break-up. This implies that, when the perturbation magnitude is a little bit smaller than the break-up threshold, the size of such structure is macroscopic.

In section 2 we recall the result by Morbidelli and Giorgilli and the main ideas of their approach. In section 3 we discuss our numerical experiments and their significance.

\section{Superexponential stability of invariant tori}

In their investigation of the dynamics in the vicinity of an invariant KAM torus, Morbidelli and Giorgilli started from the so called Kolmogorov normal form (Kolmogorov,1954).

According to Kolmogorov's construction, one can introduce suitable action angle variables $P, Q$, such that, in the neighbourhood of the invariant torus $P=0$, the Hamiltonian writes:

$$
H(P, Q)=\omega \cdot P+O\left(P^{2}\right) f(Q) .
$$

The Kolmogorov normal form shows in an equivocally way that in the vicinity of the invariant torus the significant perturbation parameter is the distance $|P|$ from the torus itself.

Therefore, in the ball $|P|<\rho$ one can introduce new action angle variables $J_{\rho}, \psi_{\rho}$ such to reduce the local perturbation to its optimal size, which, assuming analytic Hamiltonians, is exponentially small with $1 / \rho$, i.e.

$$
H\left(J_{\rho}, \psi_{\rho}\right)=\omega \cdot J_{\rho}+H_{0}\left(J_{\rho}\right)+\epsilon_{\rho} H_{1}\left(J_{\rho}, \psi_{\rho}\right)
$$

with $H_{0}$ quadratic in $J_{\rho}$ and $\epsilon_{\rho} \sim \exp (-1 / \rho)$.

At this point, it is enough to remark that, provided $\rho$ is small enough, $\epsilon_{\rho}$ is smaller than the threshold for the applicability of Arnold's version of 
KAM theorem (Arnold, 1963) in the ball $\left|J_{\rho}\right|<\rho$. This allows to prove that in the vicinity of the central torus at $P=0$ there exist an infinity of invariant tori, the volume of the complement decreasing to zero exponentially with $1 / \rho$.

On the other hand, provided $H_{0}\left(J_{\rho}\right)$ is convex in $J_{\rho}=0$, if $\rho$ is small enough, the local perturbation parameter $\epsilon_{\rho}$ is also smaller than the threshold for the applicability of Nekhoroshev's theorem. This allows to prove that the diffusion of the actions $J_{\rho}$ must be bounded by $\epsilon_{\rho}^{b}$ for all times up to $\exp \left(1 / \epsilon_{\rho}\right)$, which, by substitution gives the superexponential estimate $\exp [\exp (1 / \rho)]$. The hypothesis of local convexity is a very natural one. It means indeed that on a given energy surface, the torus with given frequency ratios is locally unique.

The picture provided by Morbidelli and Giorgilli's result is therefore the following. The tori given by Kolmogorov's theory are master tori, surrounded by a structure of slave tori, which accumulate in an exponential way to the central master torus. These slave tori are all $n$-dimensional Diophantine ones, but they are characterized by a very small Diophantine constant $\gamma$ (we recall that a frequency $\omega$ is said to be Diophantine if it satisfies the relation $|k \cdot \omega|>\gamma /|k|^{n+1}$ for all integer vectors $k$ and some positive $\gamma$ ); for this reason, they could not be found directly by Kolmogorov's construction. Moreover, diffusion among this structure of slave tori is superexponentially slow, so that chaotic orbits can enter in, or escape from, only in a time proportional to $\exp [\exp (1 / \rho)]$.

The interest of this result is double. On the one hand, this makes open the set of invariant tori from all practical point of view; this is important for what concerns the compatibility of KAM theorem with the errors in initial conditions of numerical experiments. On the other hand, a direct consequence of the local superexponential stability is that invariant tori can form, even in three or more degrees of freedom, a kind of impenetrable structure which orbits cannot penetrate for an exceedingly long time, very large even with respect to the usual Nekhoroshev's estimates.

\section{Numerical measures}

In order to test the structure of invariant tori around a chief torus we have taken as a model problem the standard map (Lega \& Froeschle 1995). We recall the set of equations for this mapping:

$$
\left\{\begin{array}{llr}
y_{i+1}=y_{i}+\epsilon \sin \left(x_{i}\right) & y \in \Re \\
x_{i+1}=y_{i+1}+x_{i} & \bmod (2 \pi)
\end{array}\right.
$$

We have computed for a set of initial conditions on the line $x=0$ the rotation number associated to each initial condition. As already pointed 

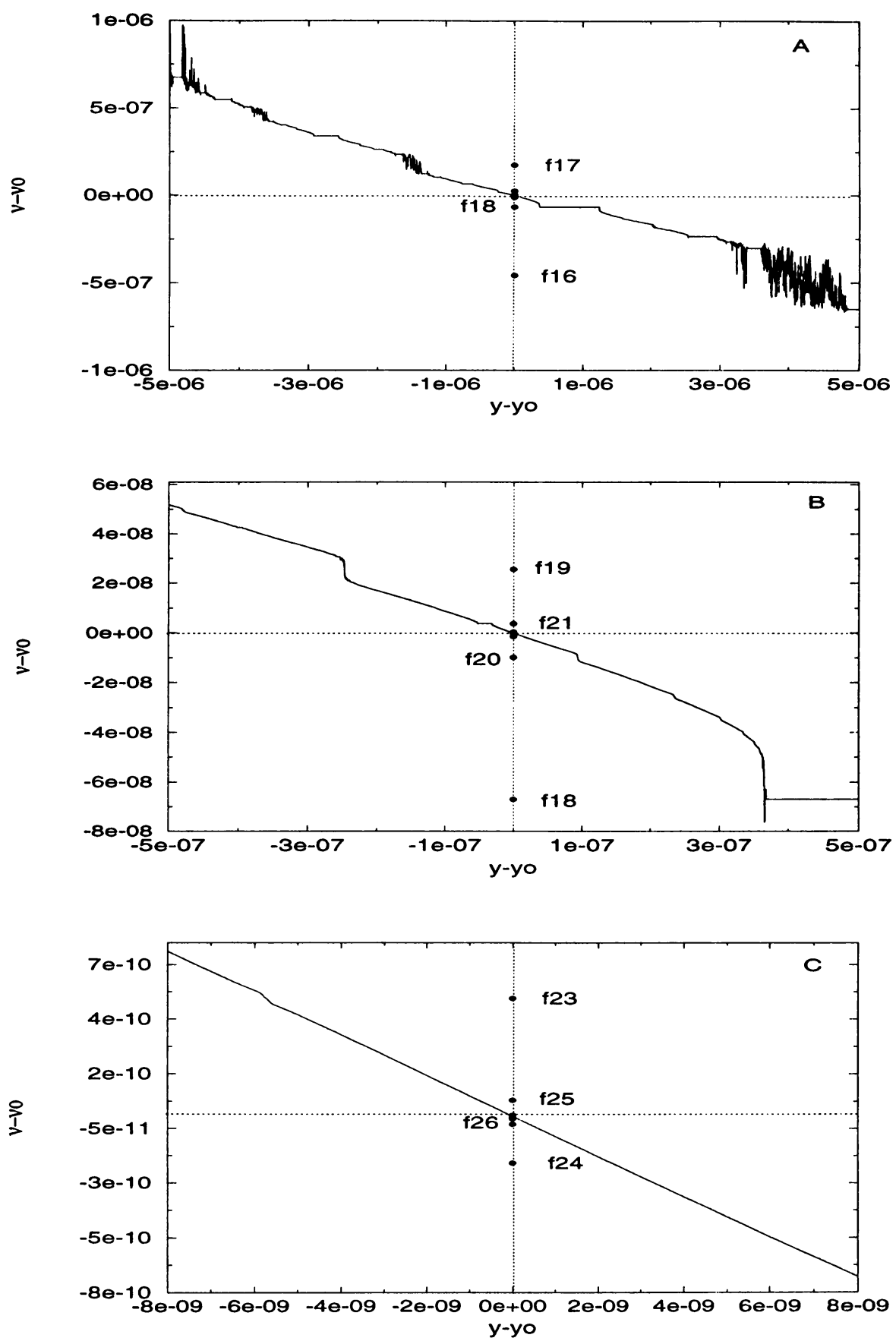

Figure 1. Variation of the fundamental frequency $\nu$ for the standard mapping, with $\epsilon=0.9715$, in the vicinity of the golden rotation number $\nu_{o}$ which corresponds to the origin of the axes. The Fibonacci terms are indicated on each figure by the set of points $f_{i}$. 

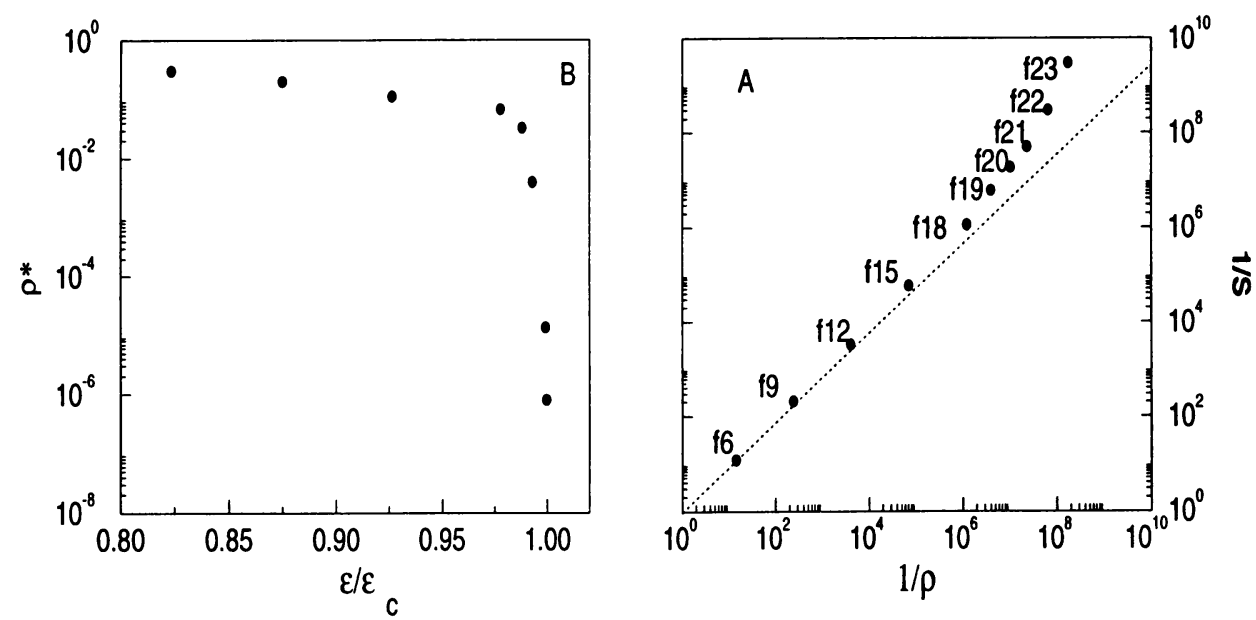

Figure 2. A) Variation of the inverse of the size $(S)$ of the Fibonacci islands as a function of the inverse of the distance $\rho$ to the golden torus. B) Variation of the threshold distance $\rho^{*}$ as a function of the perturbing parameter $\epsilon / \epsilon_{c}$.

out by Laskar et al. (1992), the existence of KAM tori corresponds to monotonic variations of the rotation number as a function of $y$. Conversely, islands correspond to constant frequencies and chaotic regions correspond to either noisy or simply non monotonic variations of frequencies. All this features appear clearly in Figure 1. On each plot the origin correspond to the golden torus, i.e. the torus whose rotation number is equal to the golden number $\nu_{o}=\frac{1}{2}(3-\sqrt{5})$. We observe that the majority of orbits of Fig.1a correspond to chaotic regions and islands. The situation changes drastically in Fig.1b: the noisy variation of $\nu$ corresponding to strong chaotic regions has disappeared, islands and crossing of hyperbolic points are still there, but their relative measure in the action variables is now definitively smaller than the relative measure of tori. This phenomenon is strongly enhanced in the last magnification: up to a step size of $\Delta y=1.610^{-11}$ (Fig. 1c), we only see one hyperbolic point and a large continuous region of tori. It is clear that the magnifications represented in Figs. 1b,c show a completely different regime and in Fig. 1c only the chief torus and its "slaves" appear with a density which seems to be in full agreement with the prevision of the Morbidelli Giorgilli theorem. We have indicated on each plot the values of frequencies corresponding to the Fibonacci sequence, i.e. the set of the successive terms obtained when developing the golden number through the continued fraction process. In order test the exponential decrease of the volume occupied by the complement of the set of tori $V_{c}$ as a function of the distance $\rho$ to the chief torus we have measured the size of the Fibonacci islands. Such islands are the largest ones and therefore they fill the major 
part of $V_{c}$.

Figures 2a show the variation of the size of the Fibonacci islands as a function of the distance $\rho$ in a $\log$-log diagram. The distance $\rho$ is the absolute value: $\left|y_{c}-y_{o}\right|$ where $y_{c}$ is the action of the center of the island and $y_{o}$ is the action of the golden torus. In the Morbidelli-Giorgilli regime we have also taken into account the hyperbolic points corresponding to the Fibonacci chain of islands. We have made an estimate of the dimension of the corresponding islands through the jump in frequency which occurs when crossing the hyperbolic point.

On Fig.2a the change of regime is drastic: at the 19th term of the Fibonacci sequence we enter in the Morbidelli-Giorgilli regime where the size of the perturbation decreases exponentially with the distance from the golden torus. Let us emphasize that the measure has been done for a value of the perturbation parameter: $\epsilon=0.9715$ very close to the critical one: $\epsilon_{c}=0.971635$.

Using the same technique of the previous we have estimated the distance $\bar{\rho}^{*}$ for a set of different values of the perturbing parameter. Fig.2b shows our final result on the variation of $\bar{\rho}^{*}$ as a function of $\epsilon / \epsilon_{c}$. After a linear decrease of $\bar{\rho}^{*}$, up to $\epsilon=0.95$, we observe a sharp drop of $\bar{\rho}^{*}$ up to $\bar{\rho}^{*}=810^{-7}$ for $\epsilon=0.9715$. It seems therefore that all the slave tori disappear at once when approaching the critical value $\epsilon_{c}$.

\section{Conclusion}

The breakthrough provided by the Morbidelli-Giorgilli theorem seems, at first glance, to be out of the range of the numerical experiments. This is still our opinion concerning the super-exponential character of the diffusion. Using the frequency map analysis we have first confirmed the existence of a chief torus surrounded by slaves. Then we have shown that such a structure exist even for values of the perturbing parameter close to the one for which no KAM tori survive. Figure $2 \mathrm{~b}$ shows the variation of the threshold distance $\bar{\rho}^{*}$ as a function of the perturbing parameter. The behavior observed for $\bar{\rho}^{*}(\epsilon)$ might deserve further studies.

\section{References}

Arnold, V. I. (1963), Russ. math. Surv., 18, 9.

Hénon, M. (1969), Quarterly of Applied Mathematics, 27, 291-306.

Kolmogorov, A. N (1954), Dokl. Akad. Nauk. SSSR, 98, 527

Laskar, J., Froeschlé, C. and Celletti, A. (1992), Physica D, 56, 253.

Lega, E. and Froeschlé, C. (1995), submitted to Physica D.

Morbidelli, A. and Giorgilli, A. (1995), J. Stat. Phys., 78, 1607.

Nekhoroshev, N. N. (1977), Russ. math. Surveys, 32, 1-65. 